\title{
Germanica
}

les arts dans les pays germanophones au $\mathrm{XX} \mathrm{X}^{\mathrm{e}}$ siècle

\section{Die Entdeckung des griechischen Mythos: Heideggers geschichtsphilosophische Wende}

La découverte du mythe grec: le tournant d'Heidegger vers une philosophie de l'histoire

The Discovery of Greek Myth: Heidegger's Turn Towards a Philosophy of History

Daniel Meyer

\section{CpenEdition}

\section{Journals}

Édition électronique

URL : http://journals.openedition.org/germanica/805

DOI : 10.4000/germanica.805

ISSN : 2107-0784

Éditeur

Université de Lille

Édition imprimée

Date de publication : 30 décembre 2009

Pagination : 13-26

ISBN : 978-2-913857-24-7

ISSN : 0984-2632

\section{Référence électronique}

Daniel Meyer, «Die Entdeckung des griechischen Mythos: Heideggers geschichtsphilosophische Wende », Germanica [En ligne], 45 | 2009, mis en ligne le 01 décembre 2011, consulté le 06 octobre 2020. URL : http://journals.openedition.org/germanica/805 ; DOI : https://doi.org/10.4000/germanica. 805

Ce document a été généré automatiquement le 6 octobre 2020.

(c) Tous droits réservés 


\title{
Die Entdeckung des griechischen Mythos: Heideggers geschichtsphilosophische Wende
}

\author{
La découverte du mythe grec : le tournant d'Heidegger vers une philosophie de \\ l'histoire \\ The Discovery of Greek Myth: Heidegger's Turn Towards a Philosophy of History
}

\section{Daniel Meyer}

Sein und Zeit verrät nirgends, daß es Heidegger auf ein Haltbares, Dauerndes, Unzerstörbares und

Bleibendes ankommen könnte, es sei denn in

Gestalt des unbedingten Feststandes der Gewißheit des Todes und also der Nichtigkeit. Es war daher nicht vorauszusehen, daß die vor dem Tode vereinzelte Existenz, an deren Endlichkeit die Ewigkeit strandet, am Ende doch noch einen „Aufenthalt" und eine „Heimat", ein „Heiles“ und sogar ein „Heiliges“ finden könnte. ${ }^{1}$

Was hier Karl Löwith mit aller Deutlichkeit unterstreicht, ist der Bruch zwischen dem Autor von Sein und Zeit und dem Heidegger der dreißiger Jahre. In einer Schlüsselstelle von Sein und Zeit, die von vielen Interpreten als Beleg für die Kontinuität in Heideggers politischem Denken herangezogen wird, bezieht sich Heidegger auf den Begriff der "Generation“ bei Dilthey. ${ }^{2}$ Dilthey sieht im Generationsbegriff eine angemessene Zeiteinheit im „Gerüst des Verlaufs geistiger Bewegungen“, „ein Verhältnis der Gleichzeitigkeit von Individuen“, wobei „die Folge der Generationen [...] ein durch Kontinuität verbundenes Ganzes“ bildet. ${ }^{3}$ Nicht zuletzt zwei vor wenigen Jahren erschienene, wichtige Texte aus dem unmittelbaren Entstehungsumfeld von Sein und Zeit erlauben es, Heideggers unausgesprochenen Bruch mit Diltheys Generationsbegriff im § 74 von Sein und Zeit deutlicher herauszustellen, ${ }^{4}$ und zwar das Manuskript „Der Begriff der Zeit" von 1924 und die Kasseler Vorträge von $1925 .{ }^{5}$ So kann im §74 von Sein 
und Zeit Zeitlichkeit, also individuelle Sinngebung, nur im Rahmen des Seins zum Tode gedacht werden, kollektive Sinngebung ihrerseits nur in einer Gemeinschaft entstehen, die jeweils synchron das Sein zum Tode erlebt, also den Gleichaltrigen, der Generation. ${ }^{6}$ Und jede Generation muss sich ihren Sinn (ob aus der Kontinuität, dem Erbe heraus oder als Bruch) neu erfinden, ihn sich wieder-holen, ${ }^{7}$ wie Heidegger dies in den nächsten Absätzen ausführt. Geschichte wird nicht mehr als Kontinuum, als ein Jahrhunderte übergreifendes Ganzes verstanden, sondern durch den Generationsbegriff in kurze Zeiteinheiten radikal abgekapselt:

Jede Zeit muß, wenn anders sie in der Eigentlichkeit ihres Seins sich verstanden hat, von „vorne“ anfangen. Je ursprünglicher sie das vermag, um so geschichtlicher ist sie. ${ }^{8}$

Wenn aber das schicksalhafte Dasein als In-der-Welt-sein wesenhaft im Mitsein mit Anderen existiert, ist sein Geschehen ein Mitgeschehen und bestimmt als Geschick. Damit bezeichnen wir das Geschehen der Gemeinschaft, des Volkes. [...] Das schicksalhafte Geschick des Daseins in und mit seiner "Generation« macht das volle, eigentliche Geschehen des Daseins aus. ${ }^{9}$

2 Sein und Zeit lässt also eine Sinngebung, die den Anspruch verträte, über den Horizont des Todes hinaus als definitives, kollektives Gründungsmoment verbindlich zu sein, nicht zu. Mit anderen Worten gibt es bei Heidegger in den zwanziger Jahren keine geschichtsphilosophische Weitenperspektive, keinen Legitimierungsprozess, der einen generationsübergreifenden Wahrheitsanspruch a priori vertreten könnte. Demgemäß verbietet es die Analyse der Geschichtlichkeit, in die Zukunft generationsübergreifend, also über zwei-drei Jahrzehnte hinaus, zu spekulieren, so dass Heideggers noch 1929 nur Spott übrig hat für solche, die - wie Spengler zum Beispiel - Geschichtsprognose im großen Stil betreiben. ${ }^{10}$ Doch genau dies tut Heidegger ab 1933 explizit in seinen Schriften und Aufrufen, insbesondere in seiner Rektoratsrede, wo es darum geht, das Wesen des deutschen Volkes im Sinne des Nationalsozialismus neu zu begründen und generationsübergreifend in die fernere Zukunft zu projizieren. Noch 1945 in seiner Rechtfertigungsschrift unterstreicht Heidegger vollkommen zutreffend diesen Aspekt:

Das Rektorat war ein Versuch, in der zur Macht gelangten „Bewegung“ über alle ihre Unzulänglichkeiten und Grobheiten hinweg das Weithinausreichende zu sehen, das vielleicht eine Sammlung auf das abendländische geschichtliche Wesen des Deutschen eines Tages bringen könnte. ${ }^{11}$

3 Es wird im Folgenden darum gehen, den Einfluss des griechischen Mythosgedanken in dieser geschichtsphilosophischen Wende zum "Weithinausreichenden“ herauszuarbeiten, die in Heideggers Denken Ende der zwanziger Jahre, Anfang der dreißiger Jahre stattfindet und sein politisches Engagement bedingt.

In Heideggers Schriften und Vorlesungen tritt der Mythosgedanke zum ersten Mal während seiner Auseinandersetzung mit Cassirers Konzept des Mythischen auf. Wie eingehend sich Heidegger damit beschäftigt, davon zeugt nicht nur eine Fußnote in Sein und Zeit und seine (im Anhang des Kant-Buchs abgedruckte) Rezension des zweiten Bandes der Philosophie der symbolischen Formen, ${ }^{12}$ sondern vor allen Dingen weite Stellen aus seiner Vorlesung von 1928/29, die eine lange Ausarbeitung der Rezension darstellen. ${ }^{13}$ Heidegger bleibt in seiner Kritik noch weitgehend dem Denken von Sein und Zeit verhaftet. Er verwirft die Idee eines Fortschritts vom mythischen zum modernen Denken, sieht vielmehr zwei verschiedene Modalitäten des Weltbezugs (im Gegensatz zur wertenden Steigerung, die im Fortschrittsbegriffs liegt, verweist der von Heidegger vorgeschlagene Begriff der "Wiederholung“ auf eine wertfreie Generationsabfolge). ${ }^{14}$ Aber in dieser Kritik des Fortschrittsbegriff, die in der Vorlesung 
von 1928/29 breit ausformuliert wird, ${ }^{15}$ schneidet Heidegger zum ersten Mal eine geschichtsphilosophische Problematik an, die ihn zunehmend zum Gedanken einer Vorrangstellung des primitiven, archaischen, von ihm als mythisch verstandenen Denkens führt - eine Vorrangstellung, die der Zeitphilosophie und der Hermeneutik von Sein und Zeit an sich widerspricht, und sich wohlgemerkt ausschließlich auf den griechischen Ursprung beschränkt. Mit anderen Worten, die Begegnung mit dem Mythischen ermöglicht es Heidegger zunächst, aus dem allzu engen GenerationsRahmen der Geschichtlichkeit von Sein und Zeit herauszubrechen und die Geschichte als einen Jahrhunderte übergreifenden Gesamtprozess zu überdenken, was Hand in Hand geht mit der Überwindung oder zumindest Ausschaltung der Todesproblematik. ${ }^{16}$ In diesen Jahren (1927-1932) lässt sich darüber hinaus eine Verschiebung von der fundamentalontologischen Gleichartigkeit zeitlicher Abfolgen (kein Fortschritt) zu einer Vorrangigkeit des geschichtlich Ersten (negativer Fortschritt) bemerken, also die Verschiebung von einem a-historisch phänomenologischen Begriff von Ursprung zu einem geschichtsphilosophischen Ursprungsbegriff. ${ }^{17}$

Diese plötzliche Erweiterung nach hinten ermöglicht erst die Erweiterung des Geschichtshorizontes nach vorne, die im nächsten Wintersemester stattfindet. Die meisten Interpreten neigen heute dazu, die geschichtsphilosophische Wende Heideggers zum „Weithinausreichenden“ (und damit 1933 zum Nationalsozialismus) in der Vorlesung „Die Grundbegriffe der Metaphysik“ vom Wintersemester 1929/30 zu erblicken. So hebt Charles Bambach einen nietzscheanisch geprägten antibürgerlichen Affekt hervor und sieht in der Vorlesung die Umschreibung eines kairologischen Augenblicks im Sinne Nietzsches. ${ }^{18}$ Doch scheint Heidegger noch zu schwanken: am Ende der Vorlesung kommt es in der Tat zu einem geschichtlichen Befreiungsgestus in die „künftige Zeit“, ${ }^{19}$ gleichzeitig unterstreicht aber Heidegger, wie noch in Marburg $1927,{ }^{20}$ die Vorrangigkeit der je eigenen Sinngebung gegenüber der kollektiven, dass die „Befreiung des Daseins im Menschen [...] jeder nur je für sich aus dem Grunde seines Wesen vollziehen kann.“" ${ }^{21}$ Doch selbst wenn Skepsis und Enttäuschung noch vorherrschen, schließt Heidegger in dieser Übergangsphase eine kollektive Sinngebung im Lichte eines geschichtlichen Ereignisses nicht mehr a priori aus:

Denn wie steht es mit unserem Dasein, wenn ein solches Ereignis wie der Weltkrieg im wesentlichen spurlos an uns vorübergegangen ist? Ist das nicht ein Zeichen dafür, daß vielleicht kein Ereignis, und sei es noch so groß, diese Aufgabe zu übernehmen vermag, wenn der Mensch nicht zuvor selbst sich aufgemacht hat, wach zu werden? ${ }^{22}$

Der Auslöser beziehungsweise die Bedingung dieser sich anbannenden geschichtsphilosophischen Wende ist nicht so sehr Nietzsche, sondern eben die Begegnung mit dem Mythischen.

7 Der zweite grundlegende Einfluss auf Heideggers Weg zum Mythologischen, und damit auch zum Nationalsozialismus, sind Walter Ottos 1929 erschienene Götter Griechenlands. ${ }^{23}$ Entscheidend ist dabei weniger die Art und Weise, wie Otto den Mythosbegriff darlegt - denn diese Darlegung deckt sich in weiten Teilen mit der Vorlesung vom Wintersemester 1928/29, was ja Heideggers Gehör für das Neue erst weckt,$-^{24}$ sondern Ottos Darstellung des griechischen Gottesbegriffs, der nicht christlich antithetisch zu Welt und Mensch, sondern in Einheit damit zu denken ist:

Das Göttliche ist die reine Gestalt der Welt, ihre Deutung, ihr Mythos. Hier kann der Gedanke gar nicht aufkommen, sich von der Welt ab und dem Göttlichen zuzuwenden. $^{25}$ 
Ottos Formel vom „Geheimnis der Berührung von Göttlichem und Menschlichen“",26 verweist schon auf Heideggers erste Hölderlin-Vorlesung von 1934/35. ${ }^{27}$ Der direkte Widerhall dieses mythischen Harmoniegedankens findet sich in der Vorlesung vom Sommersemester 1930, „Vom Wesen der menschlichen Freiheit“. Obwohl der Begriff Mythos in dieser Vorlesung nicht ausdrücklich verwendet wird, stehen Heideggers Überlegungen zur Entgrenzung auch im Kontext der Überwindung der Todesproblematik von Sein und Zeit durch den Mythos, der hier untergründig als Vermittlung zwischen Mensch, Welt und Gott fungiert:

Die Frage nach dem Wesen der menschlichen Freiheit schränkt die Betrachtung nicht nur nicht auf ein besonderes Gebiet ein, sondern umgekehrt; statt das Fragen einzuschränken, entschränkt sie es. Aber durch diese Entschränkung stoßen wir nicht von einem Besonderen her auf sein Allgemeines. Denn Welt und Gott sind nicht das Allgemeine gegenüber dem Menschen als einem Besonderen. Der Mensch ist ja nicht ein Einzelfall von Gott, so wie die Alpenrose ein Sonderfall des Wesens der Pflanze oder der „Prometheus“ des Aischylos ein Sonderfall der Tragödie.

Entschränkung führt uns in das Ganze des Seienden, Welt und Gott, inmitten dessen der Mensch selbst ist und zwar so ist, daß er zu Welt und Gott in einem Verhältnis steht. ${ }^{28}$

Dieser erstaunlich unkritische Gottesbegriff lässt sich nur im Kontext von Ottos Götter Griechenlands verstehen. Heidegger verlässt dabei nicht vollends den Rahmen der radikalen Endlichkeit von Sein und Zeit, doch wird selbst Letztere in einer parallelen, ebenfalls das Göttliche des Menschen reflektierenden Textstelle dialektisch zusammen mit einem „Auseinanderbrechen und Aufbrechen des Seienden in seiner Viel- und Andersartigkeit" gedacht. ${ }^{29}$

Die Akzentversetzung in Heideggers Denken wird besonders deutlich, wenn man Heideggers nüchterne Darlegungen über vorsokratische Denker in der Vorlesung vom Sommersemester $1926^{30}$ (also im un mittelbaren Umfeld von Sein und Zeit) mit einer Äußerung während des Sommersemesters 1931 vergleicht, wo Heidegger auf den Vorwurf, Parmenides sei ein primitiver Denker, eingeht:

In der Philosophie und so in allen letztwesentlichen Möglichkeiten des menschlichen Daseins ist der Anfang das künftig nie wieder zu erreichende Größte, das durch das Nachkommende nicht nur nicht abgeschwächt und zurückgesetzt werden kann, sondern das, wenn das Nachkommende echt ist, in seiner Größe wahrhaft groß und in seine Größe ausdrücklich eingesetzt wird. Jenes menschliche Tun aber, das auf Fortschritt sich einrichtet, dem wird freilich das Anfängliche und Frühere immer geringer und unwirklicher, und das gerade Neueste ist auch schon das Beste. ${ }^{31}$

11 Was in diesem Zitat anklingt, wird ab 1933 der Leitfaden nicht nur des philosophiegeschichtlichen (im Sinne der späteren „Seynsgeschichte“), sondern auch des geschichtsphilosophischen Denkens Martin Heideggers. Die Beschwörung des Anfangs, des griechischen Ursprungs, ermöglicht einen genuinen Neuanfang im Sinne einer Annullierung des Vorhergekommenen. ${ }^{32}$ Der Ausweg aus der Moderne, aus der Welt der Uneigentlichkeit, ist möglich durch einen Brückenschlag zum griechischen Denken. Es geht also nicht mehr darum, dass jede Generation sich ihren ihr gemäßen, authentischen Sinn erschafft, sondern darum, eine geschichtliche Krise definitiv zu überwinden. Die Möglichkeit, diese Krise zu überwinden ist für Heidegger 1933 durch die nationalsozialistische Machtergreifung gestellt, doch reiht er diesen Prozess in eine legitimierende geschichtsphilosophische Perspektive, die auf einen griechischen Ursprungsmythos zurückverweist. 
Dies wird besonders in der Rektoratsrede deutlich, wenn Heidegger die von ihm gewünschte Wende in der Universität mit dem politischen Kontext, also der nationalsozialistischen Machtergreifung durch die Wahlen (,denn die junge und jüngste Kraft des Volkes, die über uns schon hinweggreift, hat darüber bereits entschieden"), ${ }^{33}$ in Beziehung setzt. Das Verständnis für diese historische Stunde - also der Legitimationsprozess an sich (hier als Erklärung des Volkswillen) - verweist aber auf den griechischen Ursprung:

Die Herrlichkeit aber und die Größe dieses Aufbruchs verstehen wir dann erst ganz, wenn wir in uns jene tiefe und weite Besonnenheit tragen, aus der die alte griechische Weisheit das Wort gesprochen:

$\tau \alpha \ldots \mu \varepsilon \gamma \alpha \lambda \alpha \pi \alpha v \tau \alpha \varepsilon \pi l \sigma \varphi \alpha \lambda \eta \ldots$

„Alles Große steht im Sturm..." ${ }^{\text {"34 }}$

Das heißt im Sturm eines geschichtsphilosophischen Prozesses, der aus dem griechischen Denken in die überwundene oder zu überwindende Moderne hinüberweht. Dass es sich dabei in Heideggers Wunschdenken von 1933 um einen unumkehrbaren Prozess handelt, der die Zukunft definitiv verändert hat, wird in einem von Heidegger eigenhändig verfassten „Feuerspruch“ anlässlich der "Sommersonnenwendfeier" der Universität Freiburg, also einer von den Nationalsozialisten ins breitere öffentliche Leben eingeführte Veranstaltung, deutlich:

Flamme! Dein Lodern künde uns: Die deutsche Revolution

schläft nicht, sie zündet neu umher

und erleuchtet uns den Weg, auf dem

es kein Zurück mehr gibt. ${ }^{35}$

geschaffen, durch den das Volk sich wieder eine Dauer und Stetigkeit seiner Geschichte sichern soll. “" ${ }^{36}$ So dass Heidegger Ende November 1934 - im krassesten Widerspruch zu Sein und Zeit - während eines öffentlichen Vortrags in Konstanz behaupten kann: „Wer geschichtlich ist und denkt, denkt in Jahrhunderten. ${ }^{\text {"37 }}$

Diese Entwicklung lässt sich auch in seinen Vorlesungen zwischen 1933 und 1935 ausmachen. In den ersten beiden Vorlesungen vom Sommersemester 1933 und dem Wintersemester 1933/34, wo das Bekenntnis zur nationalsozialistischen Machtergreifung als heilende Revolution besonders ausgeprägt ist, hebt Heidegger das Geschichtliche des Augenblicks hervor - im Sinne einer die Zukunft des deutschen Volkes auf lange Zeit (,lang weit hinaus“"38) bestimmenden Umwälzung. Zwar unterstreicht Heidegger, dass ihm ein „prophetisches Wissen [...] verwehrt “ ${ }^{\text {“39 }}$ sei, und er distanziert sich von der Geschichts-philosophie, doch weil diese die Zukunft von der Vergangenheit (also mit ihren zu überwindenden gedanklichen Mitteln) her denkt, es Heidegger aber um einen weit radikaleren Bruch geht. ${ }^{40}$ Wie in der Rektoratsrede, aber weitaus eingehender, wird die „Weltstunde ${ }^{\text {“41 }}$ 1933, die laut Heidegger eine weltgeschichtliche Entscheidung gebracht hat, mit dem griechischen Denken in Verbindung gebracht, wobei Heidegger die Geschichte der Philosophie und die Geschichte des deutschen Volks eng verbindet. Darüber hinaus geht er so weit, auf die gleiche Abstammung der Griechen und Deutschen zu verweisen, was nicht zuletzt auch eine phylogenetische Linienführung öffnet, der sich Sein und Zeit radikal widersetzt hatte:

Wann aber und wo fiel die erste und einzige Entscheidung zur Grundfrage der Philosophie und damit zu dieser selbst? Damals, als das Volk der Griechen, deren Stammesart und Sprache mit uns dieselbe Herkunft hat, in seinen großen Dichtern und Denkern sich aufmachte, eine einzigartige Weise des menschlichen volklichen 
Daseins zu schaffen. Was da anfing, ist bis heute nicht eingelöst. Aber dieser Anfang ist noch, und er verschwand nicht und verschwindet nicht dadurch, daß die nachkommende Geschichte immer weniger seiner Herr blieb. Der Anfang ist noch und besteht als ferne Verfügung, die unserem abendländischen Schicksal weit vorausgreift und das deutsche Geschick an sich kettet. ${ }^{42}$

Die Überlagerung geschichtlicher Phasen, von Philosophie, Politik und Ethnologie, und der auf einer Beschwörung des Ursprungs ruhende seherische Gestus, reihen diesen Diskurs in ein mythisches Denken ein, das heißt in einen Legitimierungsdiskurs, der sich in Analogien bewegt, die rational nicht mehr nachvollziehbar sind, aber eben deshalb einen besonderen Wahrheitsgehalt beanspruchen. Dies wird in der zweiten Vorlesung im Wintersemester 1933/34 explizit ausformuliert. ${ }^{43}$ In seinen Vorlesungen im Sommersemester 1924 und im Wintersemester 1925/26 geht Heidegger zwar eingehend auf den Logos-Begriff ein, doch ohne jemals den Mythos-Begriff zu bemühen. Der Mythos-Begriff, den Heidegger nun einführt, entspricht exakt seiner geschichtsphilosophischen Wende, verweist er doch, im Anklang an den Anfang, als verbindliche Sinngebung in die geschichtliche Ferne:

Als $\mu v \theta$ o ist das Wort, das über den Menschen kommt, jenes, worin ihm dieses und jenes seines Gesamtdaseins gedeutet wird; nicht das Wort, in dem er von sich her Rede steht, sondern das Wort, das Weisung gibt. ${ }^{44}$

Die Philosophie selbst bewegt sich zwar im Rahmen des Logos, doch wenn es der Philosophie um Ursprünglichkeit geht, so muss sie sich sozusagen an der Grenze zum Mythos ansiedeln:

Aber der ursprüngliche $\lambda$ oүo der Philosophie bleibt dem $\mu \nu \theta 0$ ○verbunden, erst die Sprache der Wissenschaft vollzieht die Ablösung. ${ }^{45}$

Gleich am Anfang dieser Vorlesungen hatte Heidegger unterstrichen, dass die Philosophie keine Wissenschaft sei, dass also die Sprache der Wissenschaft ein ihr nicht gemäßes Medium darstellt. Heideggers Denken wird hier also zum ersten Mal implizit als Verbindung von Mythos und Logos, als Logos des Mythos, als Mytho-logos definiert. Mythologie, für Heidegger immer griechische Mythologie, ist für Heidegger 1933/34 ein Logos des Mythos, ein Prozess des Verstehens, der einhergeht mit einem Anfangsdiskurs, ein Überdenken, das zum geschichtlichen Wandel führt. ${ }^{46}$ Griechische Mythologie wird in einen geschichts-philosophischen Legitimationsdiskurs eingewoben, der die nationalsozialistische Machtergreifung auch als politische Praxis 1933/34 in ein philosophisches Großprojekt umwandelt. So wird das Verstehen des griechischen Ursprungs mit dem Begreifen, also der intellektuellen Annerkennung und somit Rechtfertigung der nationalsozialistischen Revolution und ihren politischen Möglichkeiten gleichgesetzt, umgekehrt die nationalsozialistische Gegenwart 1933/34 als mythisches Moment dargestellt:

Es ist nicht Willkür oder gar irgendein gelehrter Brauch, sondern tiefste Notwendigkeit unseres deutschen Daseins, wenn wir in diesen griechischen Anfang zurückhören.

Es bedeutet: begreifen lernen, daß jener große Anfang unseres Daseins über uns hinweg uns vorausgeworfen ist als jenes, was wir einzuholen haben, - wiederum nicht, um ein Griechentum zu vollenden, sondern um die Grundmöglichkeiten des urgermanischen Stammeswesen auszuschöpfen und zur Herrschaft zu bringen. ${ }^{47}$

Eben diese Gedankenbewegung stellt den geschichtsphilosophischen Grundgedanken der großen Hölderlin-Vorlesung von 1934/35 dar. Darüber hinaus kommt mit Hölderlin die griechische Mythologie in ihrer sprachlichen Verbildlichung zum tragen, so dass 
Heidegger ab 1934 die griechischen Götter- und Heldenwelt in seinen Vorlesungen immer wieder neu auferstehen lässt:

Gewitter und Blitz sind die Sprache der Götter, und der Dichter ist der, der diese Sprache ohne Ausweichen auszuhalten hat, aufzufangen und in das Dasein des Volkes zu stellen hat. [...] Die Dichtung ist ein Weiterwinken dieser Winke in das Volk, oder von diesem her gesehen, Dichtung ist [...] ein Zeigen, ein Weisen, in welcher Weisung die Götter offenbar werden [...]. ${ }^{48}$

Nicht nur dass Heidegger hier die Natursprache und die Sprache des Dichters als mythischen Bund zwischen Götterwelt und Volk darstellt, sondern es kommt in dieser Vorlesung zum ersten Mal zu einer Montage der Hölderlinschen Lyrik in den Heideggerschen Sprachfluss. Diese Montage wird zwar noch weitgehend durch Anführungszeichen markiert, Letztere werden aber (schon während des mündlichen Vortrages) rasch verschwinden, und nach 1936 sind lyrisch-mythologisierende Montagen für Heideggers philosophisches Sprachmaterial grundlegend. ${ }^{49}$ Hier ein Beispiel aus dem Abschluss der Hölderlin-Vorlesung:

Jenes schwer zu tragende Glück ist dem Volke dieses Landes aufgetragen: ein Zwischen zu sein, eine Mitte, aus der und in der Geschichte gegründet wird. Das kann aber nur so gesche-hen, daß dieses Volk selbst sein Dasein gründet und stiftet, d. h. erst wieder ursprünglich das Seyn nennt, dichterisch--denkerisch stiftet. So gipfelt der Auftrag und die Kunde des Adlers in der Forderung jenes dreifachen Nennens, das von diesem Land und seinem Volke, und das heißt zuerst von seinen Schaffenden, vollbracht werden muß. Zu nennen - im ursprünglich stiftenden Sagen und Wissen wieder zu eröffnen - ist einmal die Mutter, die Erde selbst. Aber eben in dieser Nennung als dichterischer tönt auf das „Vergangengöttliche“ (V. 100) in eins mit dem Zukünftigen: die Geschichte steht auf. ${ }^{50}$

Was Heidegger zitierend mythologisch kodiert, ist sein geschichts-philosophischer Grundgedanke: im ursprünglich stiftenden Sagen und Wissen - also der („eröffnenden“) Mythologie - tönt das Vergangengöttliche - also die griechische Götterwelt - in eins mit dem Zukünftigen, das heißt es vollzieht sich eine Wiederauferstehung des Ursprungs, der sinnerfüllten „Geschichte“. Dies geschieht, so Heidegger, wenn das Volk der Weisung der dichterisch-denkenden Schaffenden - also ihm selbst - folgt, so dass sich das „Wesen dieses Landes selbst findet und vollendet“, was auch bedeutet, dass es dies trotz der Machtergreifung noch nicht vollzogen hat. ${ }^{51}$

Wie sehr die neue mythologisierende Sprachlichkeit Heideggers Denken prägt, wird in der nächsten Vorlesung, der Einführungin die Metaphysik von 1935, deutlich. ${ }^{52}$ In Heideggers Kommentar des ersten Chorlieds von Sophokles' Antigone tritt das Beziehungsgeflecht aus griechischer Mythologie, Überwindung des Todeshorizonts und Politischem noch einmal besonders deutlich zu Tage. Zwischen dem Kommentar der dritten Strophe, wo Heidegger die Polis als die "Geschichtsstätte“ und dabei das Politische als an sich authentisches Moment definiert, ${ }^{53}$ und seinem Kommentar der vierten Strophe, wo Heidegger zwar die Ausweglosigkeit des Todes unterstreicht, ${ }^{54}$ was aber im selben Zuge zu seiner Überwindung im Geschichtlichen führt, ${ }^{55}$ zwischen Betrachtungen zum Politischen und zum Tod also fügt Heidegger eine „Anmerkung“ ein. Wie schon im Rahmen seiner Cassirer-Rezeption kritisiert Heidegger die Auffassung des Ursprungs als etwas Primitivem, unterstreicht die Unzulässigkeit der Volkskunde, und wiederholt einmal mehr, dass der Anfang das Größte sei und das Folgende eine „Verflachung“ darstelle. ${ }^{56} \mathrm{Er}$ schließt diese Anmerkung mit folgenden Worten: 
Die Unerklärbarkeit dieses Anfangs ist kein Mangel und kein Versagen unserer Erkenntnis der Geschichte. Im Verstehen des Geheimnischarakters dieses Anfangs liegt vielmehr die Echtheit und Größe geschichtlichen Erkennens. Wissen von einer Ur-geschichte ist nicht aufstöbern des Primitiven und Sammeln von Knochen. Es ist weder halbe noch ganze Naturwissenschaft, sondern, wenn es überhaupt etwas ist, Mythologie. -57

Das „Verstehen des Geheimnischarakters dieses Anfangs“ ist genau das, was Heidegger hier in diesem Kommentar zu Sophokles versucht, und aus diesem Verstehen leitet er „die Echtheit und Größe geschichtlichen Erkennens“ ab, das heißt in seinem Denken ab den dreißiger Jahren das Schicksal des deutschen Volkes und des Abendlandes. ${ }^{58}$ "Mythologie“ ist „Wissen von einer Ur-geschichte“ - hier gleicht Heidegger gewollt seine Definition möglichst dem normalen Sprachgebrauch an -, doch wird hier Wissen nicht im archäologisch-musealen Sinn, sondern als Prozess verstanden, auch in dem Sinne, dass Heidegger im Moment des Aussprechens gerade das leistet, wovon er spricht: Mythologie.

Und in der Tat findet der geschichtsphilosophische Mythologisie-rungsprozess, der nach dem Abschluss von Sein und Zeit eingesetzt hatte, seinen Höhepunkt. Heidegger tut am Anfang der Vorlesung gerade das, was er noch 1929 als abwegig verurteilt hatte: die Zukunft prognostizieren. Entweder geht Europa den Weg "geistigen Verfalls" weiter und es kommt zu einer „Verdüsterung der Welt" ${ }^{49}$ oder es kommt zu einem spezifisch deutschen Umschwung, „einer Erweckung des Geistes und damit [...] einer ursprünglichen Welt geschichtlichen Daseins und damit [...] einer Bändigung der Gefahr der Weltverdüsterung ", ${ }^{60}$ zu einem sdarum, die geschichtliche Gelegenheit zur Umgestaltung Deutschlands zu ergreifen. 1935 distanziert sich Heidegger - implizit von diesem Anfangsenthusiasmus, seine geschichts-philosophische Visionen bewegen sich nun im Rahmen einer (schon bei Novalis und der politischen Romantik sehr ausgeprägten) ${ }^{61}$ Kairos-Logik ${ }^{62}$ : wenn das deutsche Volk die sich ihm jetzt, also im Zuge der nationalsozialistischen Machtergreifung, bietende Gelegenheit nicht ergreift, so läutet es das Ende der Welt ein. Und die erhoffte Weltenwende schreibt Heidegger im Laufe seiner Vorlesung in den griechischen Mythos ein. Die tragische, abscheuliche Ironie dabei ist, dass diese „Verdüsterung der Welt" tatsächlich stattgefunden hat, sie aber von dem durch Heidegger verherrlichten deutschen Wesen verursacht wurde.

\section{NOTES}

1. Karl Löwith: Heidegger. Denker in dürftiger Zeit, Göttingen, Vandenhoeck \& Ruprecht, $1965^{3}$, S. 34. Vgl. auch Dieter Thomä: „Heidegger und der Nationalsozialismus. In der Dunkelkammer der Seinsgeschichte“, in: Heidegger-Handbuch. Leben - Werk - Wirkung, Stuttgart/Weimar, Metzler, 2003, S. 144-146.

2. Vgl. Martin Heidegger: Sein und Zeit, Tübingen, Niemeyer, $1993^{17}$ [SuZ], S. 384f., sowie Dieter Thomä: Die Zeit des Selbst und die Zeit danach. Zur Kritik der Textgeschichte Martin Heideggers 1910 1976, Frankfurt a.M., Suhrkamp, 1990, S. 529f. u. 543f.; Daniel Morat: Von der Tat zur Gelassenheit. Konservatives Denken bei Martin Heidegger, Ernst Jünger und Friedrich Georg Jünger, Göttingen, Wallstein Verlag, 2007, S. 113f., u. Johannes Fritsche: Historical destiny and national socialism in 
Heidegger's «Being and time», Berkeley/Los Angeles/London, University of California Press, 1999, insb. S. 139f. Insb. Letzterer unterstreicht, im Zuge Bourdieus (L'ontologie politique de Martin Heidegger, Paris, Minuit, 1988, S.67-81), die zeittypische rechtsextreme Semantik; vgl. auch Jürgen Habermas: Der philosophische Diskurs der Moderne. Zwölf Vorlesungen, Frankfurt a.M., Suhrkamp, $1993^{4}$, S. 187.

3. Wilhelm Dilthey: „Über das Studium der Geschichte der Wissenschaften vom Menschen, der Gesellschaft und dem Staat", in: Gesammelte Schriften, Band V, Leipzig/Berlin, Verlag B.G. Teubner, 1924, S. 36, S. 37 u. S. 38.

4. Vgl. Jeffrey Andrew Barash: Heidegger und der Historismus. Sinn der Geschichte und Geschichtlichkeit des Sinns, Würzburg, Königshausen \& Neumann, 1999, S. 185.

5. Wenn nicht anders vermerkt wird nach der seit 1975 bei Vittorio Klostermann erscheinenden Gesamtausgabe [GA] zitiert, hier vgl.GA 64, S. 87f., sowie Heidegger: Les conférences de Cassel, zweisprachige Ausgabe von Jean-Claude Gens, Paris, Vrin, 2003, S. 202 u. 204.

6. Hans Ruin bemerkt zu Unrecht das Fehlen im $\$ 74$ einer „geteilten Freiheit zum Tode, [...] einer endlichen Gemeinschaft, [...] einer Gemeinschaft der Endlichkeit“, denn der Generationsbegriff deckt genau Ruins Formulierungen, vgl. Hans Ruin: „Ein geheimnisvolles Schicksal - Heidegger und das griechische Erbe“, in: Heidegger und die Griechen, hg. von Michael Stegemann, Frankfurt a.M., Vittorio Klostermann, 2007, S. 24.

7. Günther Figal spricht in diesem Kontext von Enthistorisierung, vgl. Günther Figal: Martin Heidegger. Phänomenologie der Freiheit, Frankfurt a.M., Hain, 1991², S.320-325. Zu dem damit einhergehenden Problem eines Werterelativismus, vgl. Karl-Otto Appel: „Sinnkonstitution und Geltungsberechtigung. Heidegger und das Problem der Transzendentalphilosophie“, in: Martin Heidegger. Innen und Außenansichten, hg. von Siegfried Blatsche u.a., Frankfurt a.M., Suhrkamp, 1989, S. 134 f. und $154 \mathrm{f}$.

8. GA 64, S. 94. Wohlgemerkt ist hier „ursprünglich“ noch im hermeneutischen Sinne von „radikal“ zu verstehen, siehe dazu Michael Steinmann: „Martin Heidegger: Philosophie als Intensität", in: Philosophisches Jahrbuch, 2005, Nr. 112, S. 311-333.

9. SuZ, S. 384f. Hervorhebungen auch im Folgenden immer von Heidegger selbst.

10. Vgl. GA 29/30, S. 112 .

11. GA 16, S. 389f., vgl. auch ebd., S. 374, 376, 378 („Das Rektorat 1933/34. Tatsachen und Gedanken“, 1945).

12. Vgl. GA 3, S. 255-270.

13. Heidegger schickt Jaspers diese Rezension im Juli 1928, sie geht der Vorlesung also voran; vgl. Martin Heidegger / Karl Jaspers: Briefwechsel 1920-1963, Frankfurt a.M., Vittorio Klostermann und München/Zürich, Piper, 1990, S. 102.

14. Vgl. Jeffrey Andrew Barash: Politiques de l'histoire. L'historicisme comme promesse et comme mythe, Paris, PUF, 2004, S. 222f., u. SuZ, S. 51.

15. Vgl. insb. GA 27, S. 362 u. 370.

16. Vgl. GA 27, S. 381-384 (das fundamentalontologische Stichwort „Seinsproblem“ verdeckt aber noch die geschichtsphilosophische Perspektivierung, die sich anbahnt.) Löwith und Gadamer haben in der Überwindung der Todesproblematik von Sein und Zeit ein zentrales Moment der Kehre erblickt, vgl. Hans-Georg Gadamer: „Der Weg in die Kehre“ (1979), in: Gesammelte Werke, Band 3, Tübingen, Mohr, 1987, S. 276-278; Karl Löwith: Heidegger, a.a.O., S. 34. Vgl. darüber hinaus Christian Müller: Der Tod als Wandlungsmitte. Zur Frage nach Entscheidung, Tod und letztem Gott in Heideggers «Beiträgen zur Philosophie», Berlin, Duncker und Humblot, 1999, S. 262f. u. 267-269.

17. Im Gegensatz dazu rückt Lacoue-Labarthe den Ursprungsbegriff von Sein und Zeit schon in die geschichts-philosophische Perspektive eines Mythosgedanken, obwohl dieser Begriff 1927 noch völlig enthis-torisiert ist, vgl. Philippe Lacoue-Labarthe: Heidegger. La politique du poème, Paris, Galilée, 2002, S. 22-24. 
18. Charles Bambach: Heidegger's Roots. Nietzsche, National Socialism and the Greeks, Ithaca/London, Cornell University Press, 2003, S. 35f. u. 225. Vgl. auch Dieter Thomä: Die Zeit des Selbst und die Zeit danach, a.a.O., S. 501-508; Frank Edler: „Heidegger's Interpretation of the German „Revolution““،, in: Research in Phenome-nology, 1993, Nr. 23, S. 158f., sowie Marion Heinz: „Die Politisierung der Philosophie“, in: Philosophie und Zeitgeist im Nationalsozialismus, Würzburg, Königshausen \& Neumann, 2006, S. $284 f$.

19. Vgl. GA 29/30, S. 508-511.

20. Vgl. GA 24, S. $407 f$.

21. GA $29 / 30$, S. 255.

22. GA $29 / 30$, S. $255 f$.

23. Zum zeitgeschichtlichen und wissenschaftlichen Hintergrund, siehe Charles Bambach: Heidegger's Roots, a.a.O., S. 46-51 u. S. 225-227.

24. Noch 1926 sieht Heidegger in Walter Otto nur jemanden, der gegen ihn universitätspolitisch „intrigiert“, vgl. Heidegger / Jaspers: Briefwechsel, a.a.O., S. 62.

25. Walter F. Otto: Die Götter Griechenlands, Frankfurt a.M., Verlag Gerhard Schulte-Bulmke, $1947^{3}$, S. 178.

26. Ebd., S. 179.

27. Vgl. auch Otto Pöggeler: „Die kürzeste Bahn. Heideggers Weg zu Hölderlin“, in: „Voll Verdienst, doch dichterisch wohnet der Mensch auf dieser Erde." Heidegger und Hölderlin, hg. von Peter Trawny, Frankfurt a.M., Klostermann, 2000, S. 73 und 79f.

28. GA 31, S. 7f.

29. Ebd., S. 135. In dem ab 1930 gehaltenen Vortrag „Vom Wesen der Wahrheit“ kommt ein ähnlicher Gedanke zum Tragen, der darüber hinaus noch ausdrücklicher mit der Zeitlichkeit in Verbindung gebracht wird: „Die anfängliche Entbergung des Seienden im Ganzen, die Frage nach dem Seienden als solchem und der Beginn der abendländischen Geschichte sind dasselbe und gleichzeitig in einer „Zeit“, die selbst unmeßbar erst das Offene, d.h. die Offenheit, für jegliches Maß eröffnet.“ (GA 9, S. 190). Doch Heidegger lässt den Vortrag erst 1943, mehrmals überarbeitet, drucken, so dass dieser Text philologisch nicht besonders zuverlässig ist, insb. wenn es darum geht, eine Entwicklung nachzuvollziehen: was 1930 noch im Keim vorhanden ist, wurde wahrscheinlich von Heidegger so erst später ausformuliert.

30. Vgl. Franco Volpi: „Der Rückgang auf die Griechen in den zwanziger Jahren. Eine hermeneutische Perspektive auf Aristoteles, Platon und die Vorsokratiker im Dienst der Seinsfrage“, in: Heidegger-Handbuch, a.a.O., S. 36f.

31. GA 33, S. 24. Leider ist der Band 35 der Gesamtausgabe mit der Vorlesung vom Sommersemester 1932, „Der Anfang der abendländischen Philosophie (Anaximander und Parmenides)“, noch nicht erschienen. Vgl. darüber hinaus Gadamers Einschätzung, Heideggers Rückkehr zu den Griechen sei ein radikales Moment in seinem Denken: „Auf dem Rückgang zum Anfang“', in: Gesammelte Werke, Band 3, Tübingen, Mohr, 1987, S. 396.

32. Vgl. Emil Angehrn: „Die zwiespältige Entstehung der Metaphysik“, in: Heidegger und die Griechen, a.a.O., S. 193f. Zur geschichtsphilosophischen Perspektivierung des Anfangsdiskurses in der Platon-Vorlesung des Wintersemesters 1931/32, vgl. Morat: Von der Tat zur Gelassenheit, a.a.O., S. 128f. u. Bambach: Heidegger's Roots, a.a.O., S. 32, 37-39 u. 46f.

33. GA 16, S. 117. Statt der Abkapselung der Generationen wird hier auf organische Kontinuität gesetzt, so dass Heidegger ab 1933 den Begriff „Geschlecht“ bevorzugt, vgl. GA 16, S. 282 u. 306, sowie GA 36/37, S. 4 u. 89.

34. GA 16, S. 117.

35. Ebd., S. 131.

36. Ebd., S. 151. Vgl. auch ebd., S. 282.

37. Ebd., S. 324.

38. GA 36/37, S. 3. 
39. Ebd., S. 4.

40. Ebd., S. 119, vgl. auch S. $188 f$.

41. Ebd., S. 116. Man könnte nun meinen, dass Heidegger nach 1934 skeptischer gegenüber dem Nationalsozialismus geworden wäre, verkündet er doch in seiner ersten Höldelin-Vorlesung: „Die Weltstunde unseres Volkes ist uns verborgen." (GA 39, S. 50). Doch am Ende der Vorlesung heißt es: „Die Stunde unserer Geschichte hat geschlagen.“ (GA 39, S. 294), d.h. die Vorlesung hatte als klassische geschichtsphilosophische Zielsetzung, den verborgenen (hier nationalsozialistischen) Sinn der Geschichte aufzudecken.

42. GA 36/37, S. 6. Vgl. auch S. 11 und S. 89.

43. Vgl. ebd., S. 125 .

44. Ebd. S. 116.

45. Ebd.

46. Vgl. dazu auch die nächste Vorlesung vom Sommersemester 1934, GA 38, S. 132.

47. GA 36/37, S. 89.

48. GA 39, S. 31f.

49. Vgl. Erich Kleinschmidt: „Die Hermeneutik der heroischen Dekadenz. Zur Ausdruckproblematik von Martin Heideggers Hölderlin-Interpretationen“, in: Zeitschrift für Literaturwissenschaft, 1983, Nr. 51/52, S. 303-317.

50. GA 39, S. 289. Vgl. darüber hinaus, zum politischen Motiv des Schaffenden als Führer, Richard Wolin: Seinspolitik. Das politische Denken Martin Heideggers, Wien, Passagen Verlag, 1991, S. 160-167.

51. GA 39, S. 289.

52. Vgl. Kathleen Wright: „Heidegger's Hölderlin and the Mo(u)rning of History“, in: Philosophy Today, 1993, Nr. 37, S. 428f.

53. GA 40, S. $161 \mathrm{f}$.

54. Vgl. ebd., S. 167.

55. Vgl. ebd., S. 168-172. Diese komplexe Wechselbeziehung zwischen Tod, Polis und Kunst wird schon S. 139f. vorweggenommen.

56. Ebd., S. 164. Dies schon am Anfang der Vorlesung, S. 18, aber im philosophiegeschichtlichen Kontext.

57. Ebd., S. 165.

58. Vgl. ebd., S. 47.

59. Ebd., S. 41.

60. Ebd., S. 53.

61. Vgl. Ludwig Stockinger: „" Es ist Zeit ». Kairosbewußtsein der Frühromantiker um 1800”, in: Jahrhundertwenden. Endzeit- und Zukunftsvorstellungen vom 15. bis zum 20. Jahrhundert, hg. von Manfred Jakudowski-Tiessen u.a., Göttingen, Vandenhoeck und Ruprecht, 1999, S. 277-302. Vgl. darüber hinaus Philippe Lacoue-Labarthe u. Jean-Luc Nancy: Le mythe nazi, La Tour d'Aigues, Éditions de l'Aube, insb. S. 44f.

62. Siehe auch Karl-Otto Apel: „Sinnkonstruktion und Geltungsrechtfertigung“, a.a.O., S. 168, sowie, unter dem Begriff des Adventlichen, Hugo Ott: „Heidegger. Ein schwieriges Verhältnis zur Politik“, in: Heidegger. Technik - Ethik - Politik, hg. von Reinhard Margreiter u. Karl Leidlmair, Würzburg, Königshausen \& Neumann, 1991, S. $220 f$. 


\section{RÉSUMÉS}

L'article se propose d'étudier la fonction du mythe et de la mythologie grecs dans la pensée de Martin Heidegger entre la rédaction d'Être et temps et 1935. En préambule est dégagée la rupture entre la pensée de l'histoire qui régit Être et temps et l'engagement de 1933, ce qui soulève la question de l'évolution de la pensée de Heidegger. Les cours magistraux de 1927 à 1932 montrent que la pensée mythique permet à Heidegger de faire véritablement éclater l'horizon historique érigé par Être et temps et mène à un glissement de la notion d'origine vers un sens qui relève d'une véritable philosophie de l'histoire. À partir de 1933, la pensée du mythe grec est insérée par Heidegger dans un processus de légitimation, notamment à fonction politique. Ce qui apparaît dès lors dans les cours magistraux de 1933 à 1935, est non seulement le fait que le mythe grec est le cadre à partir duquel il faut comprendre l'histoire dans sa dynamique prospective, mais également le fait que Heidegger amorce lui-même un projet mythologique. Certes ce projet gagne peu à peu en autonomie par rapport aux réalisations politiques du régime hitlérien, sans pour autant qu'Heidegger ne s'en distancie explicitement.

Dieser Beitrag untersucht die Rolle des Mythosgedankens und der griechischen Mythologie in Heideggers Denken zwischen Sein und Zeit und 1935. Dabei wird zunächst der Bruch in Heideggers Geschichtsdenken zwischen Sein und Zeit und 1933 herausgearbeitet. Von diesem Ansatz aus wird Heideggers Auseinandersetzung mit dem griechischen Mythos untersucht. In den Vorlesungen zwischen 1927 und 1932 wird deutlich, dass Heidegger durch den Mythosgedanken den engen geschichtlichen Horizont von Sein und Zeit radikal ausweitet und sein Ursprungsdenken in eine neue geschichts-philosophische Perspektive rückt. Ab 1933 reiht Heidegger den griechischen Mythos in einen Legitimationsprozess ein, nicht zuletzt mit politischen Zielen. Dabei wird offenkundig, dass der griechische Mythos nicht nur den Rahmen darstellt, aus dem heraus Heidegger Geschichte in ihrer Zukünftigkeit verstanden wissen will, sondern dass Heidegger selbst ein mythologisches Projekt betreibt, das sich zwar allmählich von den politischen Verwirklichungen des Hitlerregimes verselbstständigt, ohne sich aber davon explizit zu distanzieren.

This paper addresses the part played by Greek myth and mythology in Martin Heidegger's thinking between the writing of Being and Time and the year 1935. The introduction underscores the break that occurred between the theory of history which runs through Being and Time and Heidegger's commitment to politics in 1933, thus raising a question about the evolution of Heidegger's thinking. His lectures from 1927 to 1932 show that the theory of myth allowed Heidegger to literally blow apart the narrow historical perspective set up in Being and Time, and that the concept of origin gradually took on a meaning which implies a full-blown philosophy of history. From 1933 onwards, Heidegger developed the theory of Greek myth as part of a process aiming at legitimacy, particularly with regard to politics. As a result, his lectures between 1933 and 1935 not only represent Greek myth as the frame within which history is to be understood in its prospective dynamics, but they also reveal that Heidegger himself was beginning to develop a mythological project. Even though this project gradually acquired some measure of independence from the political realisations of Hitler's regime, Heidegger never explicitly distanced himself from them.

\section{INDEX}

Mots-clés : mythologie grecque, philosophie de l'histoire 


\section{AUTEURS}

\section{DANIEL MEYER}

Université de Haute Alsace 